\title{
The Origins of Collaborative Problem Solving
}

\section{I Background}

This quasi-evolutionary account of the origins of collaborative problem solving builds primarily on research by the evolutionary psychologist Michael Tomasello (2008, 2016). It assumes that the ability to engage in collaborative problem solving is the most important reason why humans have been successful in evolution. Early humans gradually developed these skills, which made them uniquely different from other great apes. It is suggested that this process first began as closely intertwined mutual collaboration, which built on the evolution of more advanced forms of gestural communication. Three communicative motives are described, which are important in the development of the first type of collaborative problem solving. The human joy of collaboration is highlighted.

In the second part of the chapter, two antecedents to a collaborative culture are described. The establishment of a community of learners was essential in being able to transfer knowledge between individuals and across generations. Equal participation, building on reciprocity and norms, was also necessary to develop more effective types of collaboration. In the summary, this evolutionary account is compared with the modern examples of collaborative problem solving from the previous chapter.

\subsection{Antecedents to Mutual Collaboration}

Humans are different from other animals because they depend on each other in social relationships and benefit by helping each other. At some point in human evolution, the hominins who were able to collaborate gained an adaptive advantage over others. Collaboration represents a move away from great apes' total reliance on dominance as a way of settling disputes. Individuals had to become less aggressive if they were to forage together and share the spoils. Probably, ecological circumstances could 
have forced humans to forage together with a partner or else starve. It likely began with the emergence of the genus Homo around 2 million years ago (Tomasello, 2016). In this period, early humans began mating via pair bonding, which resulted in sibling recognition. When males began recognizing their offspring in the social group, they became less aggressive towards them. Humans are the only great ape that practices collaborative childcare. Individuals who are not parents will also help to feed and care for children, a tendency that may have evolved because of collaborative foraging (Harari, 20I4: IO-II; Tomasello, 20I6: 42-43).

Compared with other primates, humans are unique because they can walk upright on two legs, making it easier both to observe game or enemies on the horizon. More importantly, this permit the hands and arms to be used for a range of other purposes, like throwing stones or signaling. In this period, humans began to produce new tools. However, the disadvantage of walking upright is that the hips become narrower, which constricts the birth canal and favors earlier births. Compared with other animals, human babies are born underdeveloped and will need support for many years. Mothers could hardly forage enough food when they had needy children. It was therefore much more convenient to raise children by receiving help from other family members and neighbors. Over time, the most successful groups would be those that managed to share the spoils in a mutually satisfactory manner (Harari, 20 I4: IO-I I; Tomasello, 20 I6: 42-43).

It is likely that humans first began to collaborate in dyads or small groups through mating, hunting, or coalitionary quests for dominance. Prosocial motivation for helping and sharing with others began in mutualistic activities in which an individual who helped her partner was simultaneously helping herself. In these groups, individuals depend on each other in an immediate and urgent way, and cheating or free riding is therefore unlikely. This mutualistic collaboration is characterized by symmetrical stability and is distinctly human. It represents a move from one person dominating over another to a larger degree of complementary symmetry in doing a task (Tomasello, 2008: 7-8, I3-I6).

\subsection{Mutual Collaboration Originates from Gestural Communication}

According to Tomasello (2008), it is plausible that humans' skills and motives for shared intentionality initially emerged through mutualistic collaborative activities. When two individuals act together jointly, they naturally attend to the same situation. However, joint attention is not enough; the individuals must also know that they are attending to the 
situation together. Great apes do not engage with others in this type of joint attention, whereas human infants, quite amazingly, do it from before they are only one year old (Tomasello, 2016).

In evolution, these joint intentional activities would have started in the immediate close interaction between individuals, beginning with the gestures of pointing and pantomiming. When two individuals are working toward a joint goal in close interaction, both benefit by helping each other. Giving and receiving help will be easier when both parties engage in a closely intertwined collaborative activity. In this context, helping behavior might naturally develop as a way of facilitating progress toward a joint goal. The basic cognitive skill that is required is recursive mindreading, which implies that we both know we are cooperative.

This joint attention also makes communication toward a joint goal possible. Communicators and recipients can then interact cooperatively to get the message across. In stark contrast, two chimpanzees will never spontaneously carry something heavy together or make something together. Although apes understand that others behave intentionally according to their own goals, they do not form joint goals with others. They understand their own action from a first-person perspective and that of the partner from a third-person perspective, but they do not, like infants, have a bird's-eye view of the entire interaction. They lack an understanding of roles, which makes them unable to switch roles in an activity (Tomasello, 2008).

Young human children, but not great apes, form joint goals and take on individual roles that constitute important parts in the collaborative success. The role is impartial and partner independent; it can be applied by anyone irrespective of personal characteristics or social relationships. They communicate with each other in an attempt to coordinate the collaboration, showing that they have a "bird's-eye view" of the collaborative activity. They are able to change roles in ways that show that they are aware of individual perspectives. In the collaborative activity, it is the successful execution in itself that matters. While great apes operate according to an individual instrumental rationality, early humans were able to form the joint instrumental rationality of a pair (Tomasello, 2016).

It is proposed that vocal language first came into existence as a support to existing collaborative activities that were regulated by gestural communication. Conventional languages (first signed and then vocal) built on gestural communication. One argument that supports the view that human gestural communication is the "building block" of collaborative problem solving is that great apes have much more advanced gestural communication than vocal communication. Vocalizations are genetically 
fixed and only display specific emotions, but gestures are learned and apes can use them in a relatively flexible way in different social contexts.

However, early humans were also able to develop more advanced types of gestural communication through pointing and pantomiming. Both these gestures can provide the recipient with useful information if it is considered trustworthy. Pointing is based on humans' natural tendency to follow the gaze direction of others to external targets, and pantomiming is based on humans' natural tendency to interpret the actions of others intentionally. To communicate nonlinguistically, humans use the pointing gesture to direct the visual attention of others, and they use iconic gestures (pantomiming) to direct the imagination of others. It is likely that these two unique gestures made mutualistic collaboration possible. They arose as ways of coordinating the immediate collaborative activity more efficiently, initially by requesting that the other do something - with compliance asserted because it helped both participants (Tomasello, 2008).

Pointing is arguably the best candidate of the first gestural act that transformed humans' ability to collaborate in the immediate common ground of the mutual interaction. If we look at pointing in infants, there is evidence of a shared intentionality even before language acquisition begins (Figure 9.I). Infants are able to request things or share experiences and emotions with others. Humans are also the only primates that have highly visible eye direction, and indeed even human infants tend to follow the eye direction over the head direction of others, whereas great apes instead tend to follow the head direction. It suggests that eye contact must have had a more helpful function than a competitive function (Tomasello, 2008).

Furthermore, the communication in pointing and pantomiming is explicit, making it impossible to hide from the message without ignoring it. By letting the information "out in the open," this strengthens the interpersonal feelings of joint commitment and the trust between the parties. For example, studies of young children show that they are committed to collaborative activity through to the end; they even stay to help their partner after they have received their part of the share. When they engage in a collaborative activity, they will also be more eager to help another child, in comparison with other children with whom they have not collaborated. When the payoffs are identical, children prefer to solve the task together with others versus doing it alone. Chimpanzees do not behave like this, which suggests that human altruism toward nonfriends originates from mutualistic collaboration. The powerful interdependency mechanisms made it possible for humans to extend their sense of sympathy 


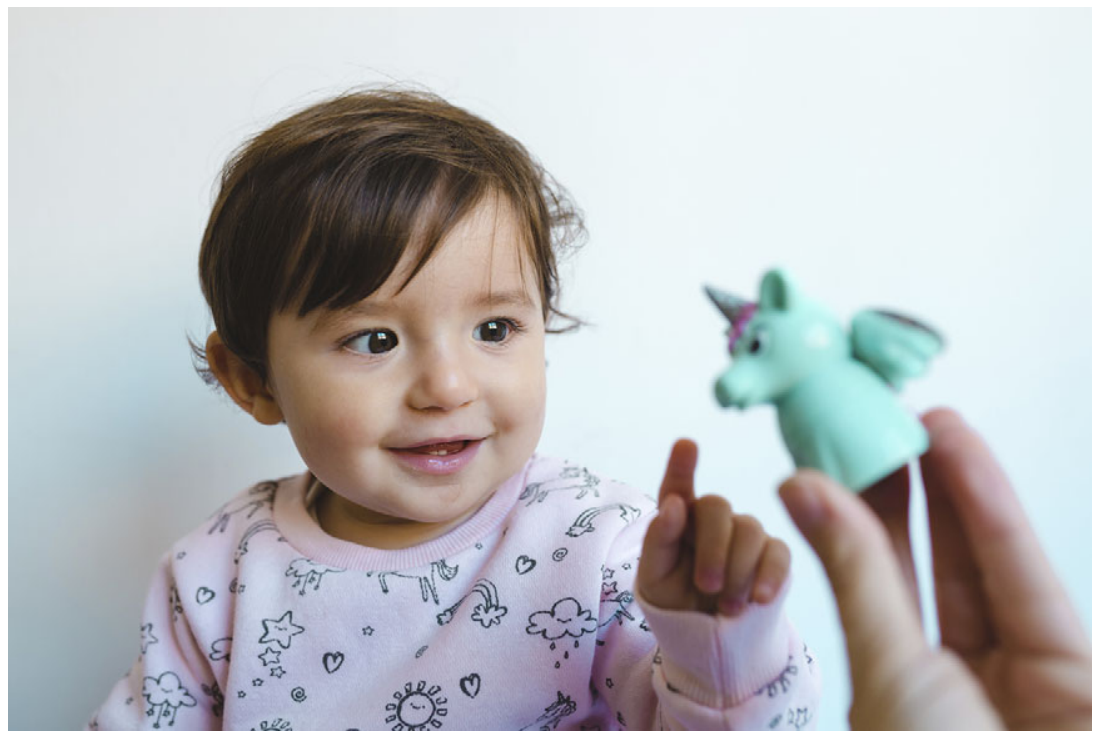

Figure 9.I Smiling baby girl pointing at a unicorn figure, photo Westend6I/Getty Images (C)

beyond kin and friends to include their collaborative partners (Tomasello, 20I6).

Is this powerful interdependency present in verbal communication too? If we fast-forward in time and look at how top solvers in innovation teams solve problems together, there are several examples that illustrate the close intertwined collaboration between individuals. One solver states, "We arrived at the solution after throwing ideas back and forth. After one member came up with a really elaborate idea we built on that and grew it into the solution." The statement suggests a process whereby ideas constantly move around in the group, being co-created and synthesized in new ways. It illustrates that verbal communication can also be a part of similar types of mutual collaboration.

\subsubsection{Three Communicative Motives}

Furthermore, Tomasello (2008: 87) proposes that the following three communicative motives were essential in the evolution of humans' unique forms of collaborative activity: 
- Requesting help or information: "I want you to do something to help me."

- Informing others: "I want you to know something because I think it will help or interest you."

- Sharing feelings: "I want you to feel something so that we can share attitudes/feelings together."

All these three motives are basic social motivations connected to helping and sharing, and they emerge early in a child's development (Tomasello, 2008: 83).

\section{Requesting Help}

The first and most obvious human communicative motive is requesting help - getting others to do what one wants them to. It is similar to intentional communicative signals that all apes have, but instead of ordering the other what to do, humans often request help. This can include hints or polite requests, but will be significantly different from ape imperatives. Since humans like fulfilling requests of others, this will often be enough (Tomasello, 2008: 84-85). In collaborative problem solving, requesting help will be valuable in many different ways. If we look at verbal communication, a top solver in a virtual innovation team illustrates that naive questions are valuable for all parties:

The team interaction is interesting because the other folks on the team did not have the same kind of technical background. So their naivety or their lack of experience allowed them to ask questions and maybe even question paradigms that someone who does have the technical background would not do. And I saw value in that.

This statement underlines the value of unexpected questions from individuals who lack experience, but still bring in more cognitive diversity. It shows that help is not only about transmission of information, but it can challenge the helper to rethink his own perspectives.

\section{Informing Others}

The second uniquely human communicative motive is to help others by informing them of things they find useful. Here, the gesture of pointing is limited because it cannot inform about things displaced in time and space. Instead, iconic gestures like pantomiming are more effective because more information is present in the gesture itself. Sometimes, individuals will even offer help to others when the information is perceived as irrelevant by the recipient (Tomasello, 2008). When informing others through verbal 
communication, the communicator can also use this as a strategy to "think aloud." For instance, a solver in an online team illustrates the importance of expressing unfinished thoughts:

I think explaining your reasoning out loud to somebody else is an incredibly good way of deciding whether there's a basic flaw there. When you talk out loud you certainly hear yourself and say, "I'm not saying that, am I?" whereas if you think it, it sounds perfectly reasonable. So I don't think it's really very different.

The solver explains how the act of informing can help detect flaws in your own thinking. Informing others is not only helpful for the recipient, but also for the communicator. Thinking aloud will typically emerge as an important element in spontaneous discussion in dyadic collaboration (Baltzersen, 2017).

\section{Sharing Feelings}

The third basic communicative motive is an expressive or sharing motive that refer to people simply wanting to share feelings and attitudes about things with others. It can be a child who points to a dog to share the enthusiasm for the dog. It expands the social bonding with others and strengthens group membership. In verbal communication, it is present through gossip about all kinds of things (Tomasello, 2008). In this type of motive, it is also important to cope with negative feelings. A solver in an online team exemplifies the importance of critique:

For me, it is more accurate to say that I don't necessarily have a clear idea of the solution when I start, or if I do it often changes. Sometimes, you may be in love with the first thing that comes into your mind and you say to yourself 'Oh, I'm so brilliant?. But you have to be critical of yourself as well and try to find the holes in it. I have done one challenge on my own and the rest have been in a team environment. One of the values of working in a team is the critique. It is better to hear the critique from your colleagues before you submit a solution than hear the critique from the seeker.

Here, the solver highlights the value of giving each other critical feedback in the teams.

\subsubsection{The Joy of Collaboration}

All three communicative motives assume that getting the message across will be mutual beneficiary for all parties involved. If a human communicator requests help (all other things being equal), the recipient will want to 
help, and if the communicator offers information, it is mutually assumed that the information is useful. Finally, if the communicator wants to share attitudes, they assume that the sharing will be interesting to the recipient. When the communicator overtly signals his intention to communicate, both parties try to ensure that the communicative act succeeds (Tomasello, 2008: 88-9I).

A fascinating consequence of these communicative motives is that the collaborative activity is often in itself perceived as rewarding. In one interesting experiment, children between one and two years old were compared with juvenile human-raised chimpanzees on four collaborative tasks. Two tasks had a specific goal, and the two others were social games without a goal other than playing the collaborative game itself (e.g., the two partners using a trampoline to bounce a ball up in the air). The human adult collaborative partner was instructed to stop doing anything at some point to determine the commitment to the joint activity. The results showed that the chimpanzees were able to synchronize their behavior relatively skillfully in the instrumental tasks, but showed no interest in the social game. Most interestingly, when the human partner stopped participating, the chimpanzee never made a communicative attempt to reengage the partner even when they had previously been highly motivated in the instrumental tasks. They only participated in the tasks in an individualistic manner. In contrast, the human children collaborated in the social games and they even transformed the instrumental tasks into social games by placing the obtained reward back into the apparatus to start the activity again. It showed that the collaborative activity itself was more rewarding than the goal. When the adult stopped participating, the children actively sought to reengage the person, suggesting that they had a shared goal (Tomasello, 2008: 177-178).

If we look at modern examples of mutual collaboration, several of the top solvers are also motivated because they enjoy the teamwork. One solver states:

It was extremely stimulating and it pushed me to seek and elaborate information and knowledge that otherwise I would not have sought. Working on and building on the ideas of other contributors was extremely enjoyable. The plurality of perspectives on a certain idea can open new directions of thoughts and, ultimately, stimulate the creativity.

This solver underlines the joy of "building on the ideas of other contributors." It illustrates how motivation is closely connected to the coconstruction of new and unexpected thoughts. Another solver even 
expresses the paradox of enjoying the uncomfortable, "So it supplements your knowledge with other people's knowledge. You work with people that are out of your comfort zone which I really enjoy because it pushes you to do more research into a challenge and push back against other people and really make innovative kinds of solutions." The examples illustrate the positive feelings that emerge through the collective work in itself.

\subsection{Antecedents to Collaborative Culture}

How were humans able to extend beyond mutual collaboration and create collaborative cultures that permitted transfer of knowledge across generation? If we compare chimpanzees and humans, a major difference is that chimpanzee groups are not able to accumulate knowledge over time. In contrast, humans use cultural artefacts and engage in practices that other humans have invented before them. This learning across generations opens up for further improvements and refinement of artifacts and practices (Tennie, Call, \& Tomasello, 2009). In this part, I examine two core components that enable such a collaborative culture.

(I.) A community of learners who utilize observational learning (social learning) and explicit teaching

(2.) Equal participation (equal sharing)

In combination, it is assumed these unique processes enabled the cumulative cultural evolution of knowledge across generations (Tennie et al., 2009). These issues will be further explored in the following sections.

\subsection{The Emergence of a Community of Learners}

When did we as humans become a community of learners? Researchers claim that our evolutionary story as tool users can provide some degree of answer to how this happened. Stone toolmaking (knapping) is a complex skill integrating demands for planning, problem solving, and perceptualmotor coordination within a collaborative social context (Pargeter, Khreisheh, \& Stout, 20I9; Stout \& Hecht, 2017). However, if we look at the first stone toolmaking which began approximately 2 million years ago by Homo erectus, this knapping only involved simple hammer techniques that required less demanding manual skills. Homo erectus had adjusted to the upright walking position and could use their hands in completely new ways, but stone tools were still used in a simple way for a 


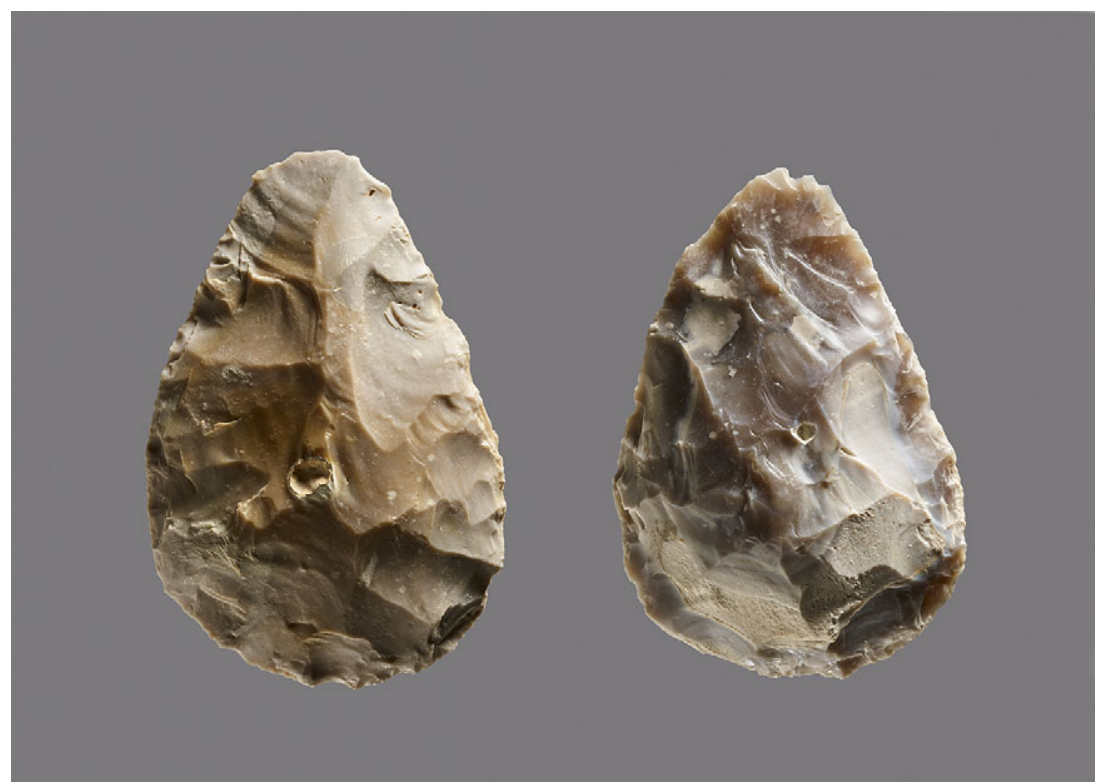

Figure 9.2 Regular flint handaxe from Boxgrove, West Sussex, England. From the Acheulian period. The typical tool is a general-purpose handaxe. Credit: (C) The Trustees of the British Museum

long time. Therefore, it was not necessary to invest much time in skill learning (Pargeter et al., 20I9; Stout \& Hecht, 2017).

However, about 500,000 years ago, skill-intensive biface-thinning techniques emerged, providing powerful evidence of a new capacity of learning among early humans. Boxgrove, UK is one of the richest and the oldest handaxe sites in Europe (dated ca. 524-478 BC) and it provides evidence of a handaxe production with smaller, thinner, more regular and symmetrical forms (Figure 9.2). The cores and flakes have been carefully shaped, revealing the use of knapping techniques such as soft-hammer, percussion, and platform preparation that are comparable to how modern experimental knappers work (Pargeter et al., 2019; Stout \& Hecht, 2017).

Knapping is a practice which removes flakes from a stone core by using precise and controlled ballistic strikes with a handheld hammer (typically stone, bone, or antler). Only a small error in the strike will ruin the process. Expert knappers need to possess complex perceptual-motor skills, understanding the relationships between the force and location of the strike and how to position the core. Such a skill must not only be executed, but also observed and evaluated (Stout \& Hecht, 20I7). 
Furthermore, training time is essential. Paleolithic foragers would have had to balance the costs and benefits of making and maintaining technology against the need to find food, avoid predators, and reproduce. Tool production time and foraging efficiency were obviously important factors to consider, but one study highlights instead the costs of skill acquisition as another important factor. In the experiment, modern participants were trained to make stone tools. The study shows that $\sim 200$ hours of deliberate practice is required for refined handaxe production. The knapping learning curve follows a well-known "power-law of practice" that is common in both informal (sewing and cooking) and formal (biology and chess) learning. There were rapid initial increases in knapping skill followed by diminishing returns as performance approaches a local optimum. Although 200 hours in total might not appear to be a long time, other extra activities like the preparation of raw materials (e.g., spalling) and knapping tools (e.g., billet production) also had to be done (Pargeter et al., 2019).

Knapping is more of a flexible skill than one specific type of action. One needs to learn how to link effective means to appropriate goal-orientated action in many different ways depending on the specific task. In comparison, it is much easier to learn Oldowan knapping because it allows for more errors (Stout \& Hecht, 20I7). Learning the skill also requires extended investment in deliberative practice, directed toward improving performance through sustained effort and attention despite setbacks and frustrations. It requires discipline and self-control and is not necessarily enjoyable or rewarding in the short term. Learners must continuously check the actual incorrect outcome with the predicted outcome, and engage in a lengthy process of behavioral exploration to assess task constraints and refine skills. The largest neural and cognitive demands do not occur during the expert performance of the stone tool, but instead during the process of learning how to make stone tools. The working memory is taxed more heavily during the acquisition of expertise (Ericsson, Krampe, \& Tesch-Römer, 1993; Pargeter et al., 2019; Stout \& Hecht, 2017).

There is no doubt that making late Acheulean style handaxes requires both time and effort and a certain level of cognitive and affective learning. In addition, if the skills are only mastered by a small percentage of the group, they are vulnerable to loss. If the learning costs are high, it is less likely that others will acquire such skills. However, in a culture of teaching and learning, such a complex skill would more easily be maintained. The skills of making these tools suggest that hominid cognitive and 
technological complexity was going through a major transformation in this period. It suggests the presence of what could be the first advanced community of learners. Knapping skill acquisition involves the copying and high-fidelity production of stone tools, and probably required a community that encouraged collaboration, sharing of knowledge, and intergenerational reproduction of complex skills (Pargeter et al., 2019; Stout \& Hecht, 20I7).

If humans began to teach each other how to use these stone tools in this community, it is also likely that they developed the first human culture (Pargeter et al., 20I9; Stout \& Hecht, 2017). In general, explicit teaching is considered to be essential in cumulative cultural evolution. Teaching is present in all human societies, but it is not a common activity among chimpanzees or other nonhuman primates. Both children and adults are sensitive to teaching in their imitation of others. Teaching also involves a certain degree of altruism, in that the adult instructor needs to spend time and energy to ensure that a child acquires certain skills or knowledge. Children automatically trust adult teachers and are eager to change their behavior, in a way that chimpanzees apparently are not (Tennie et al., 2009).

Archaeological evidence cannot demonstrate a particular form of teaching, but the knapping skill requires the use of complex techniques that even modern humans will struggle with if they do not receive explicit instruction. The tools provide evidence of a more complex learning and teaching practice that involved both individual practice and social support (Pargeter et al., 2019; Stout \& Hecht, 20I7). Instruction could have been given as intentional demonstration, communicative gestures, or some type of linguistic instruction. As in apprenticeship learning or coaching, skill acquisition practices involve a combination of social learning opportunities like observation, instruction, and motivated individual practice (Stout \& Hecht, 20I7).

If we compare humans with apes, an important difference is that humans are able to learn socially of the actual actions performed by others (process copying), not just the results produced on the environment (process copying). Humans are effective in copying others' behavior and this begins early with the infant who imitates mom when observing her. Children do not only imitate to acquire more effective behavioral strategies in solving instrumental problems, but they also imitate for purely social reasons. In acquiring linguistic conventions, children are not only motivated by communicative efficacy, but by a desire to do it in the same way as others do. They conform to the group and imitate others simply because they want to be like them. The evolutionary basis is very likely 
identification with the group, motivating conformist cultural transmission and more faithful reproduction of behavior. In modern humans, one example is our tendency to follow fashions for no apparent instrumental reason (Tennie et al., 2009).

In contrast, chimpanzees learn how or where a box works, but they are not attentive towards the actions or the behavioral techniques that are used. For example, when chimpanzees observe someone using a tool, they tend to focus on the effect being produced in the environment, but they pay little attention to the actual bodily actions of the tool user. Instead, they use their own behavioral strategies with the goal of producing the same environmental effect. Thus, they reconstruct the product rather than copy the process leading to it. They solve problems by themselves and are reluctant to adopt any new behavioral strategy if they already have one that works. Consequently, the cultural traditions of nonhuman primate species do not seem to accumulate modifications over time. Chimpanzees are in a way reinventing the same wheel again and again through emulation learning (Tennie et al., 2009).

Humans are different since they can pay attention to the actual behavior or behavioral strategies of a demonstrator, and these processes must have been very important among the community of learners in Boxgrove. As part of a toolmaking practice, one type of observational learning would likely have aimed to copy the observed actions of others through a process of matching or "motor resonance." This requires the ability to translate visual and auditory information of another's actions to appropriate motor commands for one's own bodily actions, probably also attempts to copy bodily postures and gestures. This skill learning requires a significant level of general intelligence since a number of subtasks must be organized into a coherent mental program (Stout \& Hecht, 20I7). This copying of processes also enables the further modification and improvement of artefacts and practices across generations (Tennie et al., 2009).

\subsubsection{Equal Participation}

Obviously, there will be norms present in a human collaborative culture. Adults will expect that children behave in certain ways. Children do not only understand that something is done in a specific way, but also that this is how things "should be done." At some point in children's development, they expect that other persons ought to respond or help as requested, and they become offended if this does not happen. They begin following 
norms that regulate social behavior. In contrast, nonhuman primates show no signs of making normative judgements. Nonconforming behavior is not punished. In contrast, even three-year-old human children object if others do something the "wrong" way, and teach the offender how to do it the "right" way. This normative dimension does not only arise from explicit teaching, because when children observe adults, they will often think that everyone else should follow the adult behavior shown to them. This normative dimension of social learning strengthens the faithful transmission of knowledge skills across generations, reinforcing group identity and conformist transmission (Tennie et al., 2009; Tomasello, 2008: I33).

An important question is how sanctions and social norms have evolved. Mutual expectations of helpfulness are not norms because they have no punitive force, but they are one step in that direction (Tomasello, 2008: 208). Tomasello (2016: 49-50) claims that the emergence of a sense of fairness and justice originates from the joint intentionality as effected by a collaborating dyad. To coordinate collaborative activities, humans evolved skills that enabled them to form joint goals. Both the self and the other were recognized as important in the collaborative enterprise, and this mutual respect led to the emergence of genuine morality. In this new cooperative rationality, it made sense to depend on a collaborative partner. Individuals who were able to act together dyadically as a joint agent "we" were also able to structure their individual roles and perspectives. Over time, they developed a common ground understanding of ideal roles such as in stone knapping teaching. This practice eventually evolved into socially shared normative standards that specified what either partner must do in their specific role of being a teacher and a learner (Tomasello, 2016).

Reciprocity occurs widely in nature, but there is a difference between emotional (or attitudinal) reciprocity and calculated reciprocity. Mammals and especially primates, show emotional reciprocity because of their ability to form long-term emotion-based social relationships. Chimpanzees show positive affect toward those that help or share with them and with whom they engage in long-term social relationships. They can also feel sympathy for each other. Individuals form emotional bonds with those who help them and then they naturally help kin or "friends." The origin of this type of reciprocity is probably based on offspring bonding to those who succor or protect them. However, this does not explain the mechanisms that lead individuals to form friendly social relationships with nonkin.

Calculated reciprocity is a very different type of reciprocity, building on an implicit contract or rule like "we each keep track of who has done what for whom and stop cooperating if we are giving more than we are getting." 
If I help you on one occasion, you will help me on the next occasion, as far as we benefit of it in the long run. This classic tit-for-tat reciprocity requires that we obligate ourselves to a future course of action, which only humans are capable of. Except long-term social partners, there is very little evidence that great apes engage in any exchange of favors. For instance, one experimental study found that randomly paired chimpanzees did not preferentially help an individual that had just helped them over one that had not (Tomasello, 20I6).

This morality of fairness is confined to the human species. It is characterized by a sense of responsibility or obligation: "I do not only want to be fair to all concerned, but one ought to be fair to all concerned." Collaborative partners will be accountable for their actions by invoking interpersonal judgements of responsibility, obligation, commitment, trust, respect, duty, blame, and guilt. Humans also show resentment or indignation against unfair others. In contrast, great apes do not appear to have a sense of fairness in dividing resources, and they exhibit no sign of so-called retributive justice. Nor was free riding any problem initially among early humans. The number of individuals available was the same as the number needed for foraging success, but at some point, they would not allow others to get the spoils.

The simplest way of sharing is to let participants get equal shares and nonparticipants get nothing, and indeed, young children have a very strong tendency to divide the spoils of a collaboration in this way. Studies even show that young human children, but not great apes, share the spoils of a collaborative effort even when one child is given the opportunity to take everything. Young children also modify their own cooperative behaviors depending on whether others are watching. One explanation can be that early humans' collaborative activities took place in the context of partner choice in which potential partners evaluated others for their cooperativeness.

We also know that almost all contemporary hunter-gatherer groups are highly egalitarian. Dominant individuals are quickly overrun by a coalition of other individuals who are superior because of their group size. In a group hunt, the catch is almost always shared with others, not only in the immediate families, but more broadly in the social group at large. These social norms are usually very strict, and sanctions are used if they are not followed (Tomasello, 2016).

In contrast, the chimpanzees act according to the principle of "first come, first served," not equal sharing. The logic behind "tolerated theft" among chimpanzees is that if the possessor chooses to fight the harasser for 
the meat actively, he will likely lose more of the food to others nearby who will continue eating. The best strategy is to eat quickly all that he can, and allow others to take some meat to keep them happy. Hunters will obtain more meat than latecomers because they are the first at the carcass (Tomasello, 2008: I83). If there is a competition over food resources, collaboration will often fail. In one experiment, a pair of chimpanzees were presented with out-of-reach food that could only be obtained if they each pulled on one of the two ropes available simultaneously. First, when there were two piles of food, one in front of each participant, there was a moderate amount of synchronized pulling. However, when there was only one pile of food in the middle of the platform, making it difficult to share at the end, coordination fell apart almost completely. These findings demonstrate that chimpanzees are only able to synchronize activities when there is no quarreling over the food at the end. Although chimpanzees sometimes help humans, they do not help others if they themselves have a chance to obtain food (Tomasello, 2008: 183-184).

If we look at our human history, the arguably first type of formalized collaboration that emphasized equal participation were the collegial boards in ancient Athens. In the fourth century BC, the Athenians began to annually select some 700 magistrates. A lottery picked 600 hundred of them from the citizenry at large, while only roo were elected in the Assembly. Most of these magistrates served on collegial boards, typically comprising ten persons with one representative from each tribe. All members were on equal footing, and there was no formal leader of the group. Decisions were based on discussions amongst the members, and if they disagreed there would be a vote and the majority decision bound all. These magisterial boards worked with public affairs according to the board's constitutional charter, such as leading armies, maintaining oversight of public festivals, and disbursing welfare payments. Service on most teams was intense for its duration, but it was limited to a single year. This reduced the risk of harmful strategic behavior. Selection by lot guaranteed that team members would bring in a large variety of different personal perspectives. Together with a background culture that emphasized formal equality in respect to public speech and vocal dissent, these features would likely create conditions that allowed for equal participation through the inclusion of all group perspectives (Hansen, I99I: 237; Ober, 2008).

Even today, public discussions in democracies are reliant on equal participation. Because these conversations invite to conflict and can be deeply uncomfortable, they require formal and informal rules of 
engagement. According to Schudson (1997), what makes conversation democratic is not free or spontaneous expression, but equal access to the floor, equal participation in setting the ground rules for discussion, and rules designed to encourage focused talk. The insistence on equality and a social order that creates a certain level of publicness are core components in the democratic conversations.

The emphasis on equal participation and debate is also an important part of modern collaborative problem solving in virtual innovation teams. A top solver in a team explains:

We sometimes have to go through a lot of argument, a lot of debate. I remember I was in agreement with another solver but the other two were not in full agreement, and everyone had to make their case as best as they could in order to convince the rest of the team. I think that was really challenging.

The disagreement was solved by letting everyone make their case in order to convince the rest of the team. In the interview, this solver follows up and underlines the learning value of the process ("But I believe the amazing thing is that we learned a lot") and the individual effort that members put into such type of work ("you try to come up with the best out of yourself.")

If we look back at the magisterial boards, some historians claim that as many as ten individuals were selected just in case somebody turned out to be incompetent or unreliable. Although some individuals must have been incompetent, there are surprisingly few examples of complaints or people actually being dismissed. Moreover, when a magistrate was brought into court, the charge was usually bribery or corruption, not incompetence. According to the sources, the administration appeared to have worked satisfactorily in the lottocratic system with the support of a small group of clerical staff. Because the lottery was voluntary, candidates would usually be motivated to work in the administration, and the tasks would typically not require a high level of specialist skills (Hansen, I99I: 238-244).

The tasks in the board were based on the laws and decrees, and the magistrates were accountable for the funds they had used. Accountability procedures were strict, and all of them were assessed on reputation for character and conduct of life, rather than competence. Before leaving office, the magistrates also had to undergo a formal review (euthuna) of their work during the year. A special emphasis was put on the public funds (Hansen, I99I: 238-244). Gaining a good reputation as collaborators has probably been an important motivation early in evolution, too (Tomasello, 2008: 324-325). 
Furthermore, even in virtual innovation teams, solvers highlight the value of being among "equals":

There is ego, but it doesn't feel like you are in a workplace because we're not climbing a ladder. We all receive the same reward for a successful solution, the recognition is the same. So there is no distinction for the reward. That's actually the perfect way to do it. As equals, all of our names go on the final proposal. So there's no way to stand out amongst your peers in that team.

The solver highlights the importance of receiving the same recognition for the group work. The description of being equals is contrasted to workplaces where there is a lack of a symmetrical relationship.

What appears important in all types of equal participation is being able to tackle disagreements and still acknowledge each other. The magisterial boards were organized to encompass these tensions, and so do modern innovation teams. A top solver illustrates this attitude when he is asked whether he is bothered by his ideas being critiqued:

That's a fine line. Your ego can suffer because you might think to yourself, "OK, the other team members don't value me or don't value my solution." But if people are conscious of these feelings and provide clear argument that comes from a sound base, then we can tolerate criticisms. We are rational people in this industry. If it's just hand waving and you say it's not going to work and don't tell me why, then I might get offended.

In this type of collaborative problem solving, it is vital to accept critique and not think that this means that your opinions are unappreciated. If proposals in the group are refused without argumentation, it may create negative feelings. Interestingly, the emphasis in collaborative problem solving should be on the quality of the arguments and not the persons making them. It indicates the importance of having discussion rules that can control emotions and enable the best argument to win through a fair and open discussion.

\subsection{A Summary of the Evolution of Collaborative Problem Solving}

The evolutionary analysis of collaborative problem solving in this chapter points to two distinctly different subtypes of collaborative problem solving. First, mutual collaboration points to what can be labeled as elaborative collaborative problem solving, building on the sympathy between collaborators and the presence of immediate helping behaviors like request, informing, and the sharing of feelings. In this type of collaboration, embodied interaction will be important. There is also a joy of being part 
of the collaboration in itself. With the flexibility of verbal language, this collaboration moves forwards as a spontaneous, rapid and open dialogue where individuals co-construct thoughts and think aloud. In general, the elaborative aspect of this type of problem solving does not follow a strict organization of the collective work, but members can join or interrupt conversations at any time in a more flexible way (Baltzersen, 2017). The stories from top solvers illustrate how different types of elaboration play an essential role in innovative problem solving. As the solver stories show, the open exchange of ideas enables persons to build on each other's thoughts and trigger them to move the discussion forward. In this intertwined collaboration, individuals both make requests for help and inform each other, in ways that are beneficial for both parties. In this context, help will also be related to how different tools mediate and display shared information in a common working space (Baltzersen, 2017). These group processes should be examined in more detail to better understand CI.

Second, the evolutionary analysis of collaborative culture points to the emergence of what can be labeled as rule-governed collaborative problem solving. It is likely that the transition from mutual collaboration to collaboration in larger groups involved the invention of norms. As mentioned by Tomasello (2016: 2, 9-10), the dilemma is that in most situations that require fairness, there will typically be present a complex interaction of the cooperative and competitive motives. On one hand, sympathy can be regarded as pure cooperation building on mutual interdependent collaboration. On the other hand, fairness represents a cooperativeness of competition in which individuals seek balanced solutions to the many and conflicting demands of multiple participants' various motives. Finding a satisfactory balance between cooperation and competition is the basic challenge of a complex social life. Competition is related to individuals' power and dominance in contesting resources. It requires some type of contract or rule to be solved. Therefore, the morality of fairness is much more complicated than the morality of sympathy (Tomasello, 2016: 2, 9-10).

In this quasi-evolutionary account, it has been suggested that rulegoverned collaborative problem solving emerged through two key practices, skill acquisition and sharing of food. The findings at Boxgrove show that collaborative cultures may have emerged very early in human history. Stone tool learning required deliberate practice, with a minimum of 200 hours training. This skill acquisition is more cognitively demanding than the expert performance in itself. Individual training, observational learning, and explicit teaching would probably have been necessary to 
acquire the necessary skills. In this community of learners, tools would have been refined and improved over time and across generations.

Second, it is likely that hunter-gatherer groups were important in the development of equal participation as a social norm. Equal participation, with its emphasis on calculated reciprocity, represents a significant move away from the dominance of a few individuals in groups. From an evolutionary perspective, equal sharing of food required increased control of emotions and the establishment of norms that kept free riders out. A fair sharing of spoils also opened up the possibility of greater role differentiation in groups; not everyone had to participate in the hunt.

The democracy in ancient Athens was one of the first institutions that formalized equal participation as an important principle in collaborative problem solving. The collegial boards of magistrates were chosen by lot. It ensured both a fair selection and a large degree of cognitive diversity. This diversity of perspectives increased the likelihood of utilizing all relevant contributions. The conversational culture in Athens also allowed citizens to be critical and discuss uncomfortable topics.

Even today, the CI research still underlines the same principle of equal participation as a key success factor. This is important not only in innovation teams, but also in group discussions in Deliberative Polling. As mentioned by Schudson (I997), a democratically oriented conversation is not primarily spontaneous or free, but essentially rule-governed; civil discussion is guided by a conception of equal participation.

Chapter 6 outlined three key topics in CI research related to collaborative problem solving: $\mathrm{I}$. Working well with others, 2. Cognitive diversity, and 3. Group organization. Table 9. I gives an overview of how these three topics connect to elaborative and rule-governed collaborative problem solving.

First, regarding the ability to work well with others, the analysis shows that rule-governed and elaborative collaborative problem solving build on different types of morality. Both create a symmetrical relationship, but while elaboration centers on interactional symmetry, the rule-governed approach seeks equal participation. Since elaboration builds on close interthinking, there is present a strong degree of mutuality when individuals build on each other's ideas in the ongoing talk. This leads to the establishment of a shared understanding, or a "we," which dissolves the separate individual positions. This interactional symmetry creates sympathy, which therefore increases the acceptance of unequal contributions. In rule-governed collaboration, the morality of fairness demands that everyone adheres to the shared collaborative norms. 
Table 9.I. A comparison of elaborative collaborative problem solving and rule-governed collaborative problem solving

\begin{tabular}{|c|c|c|}
\hline & $\begin{array}{l}\text { Elaborative collaborative problem } \\
\text { solving }\end{array}$ & $\begin{array}{l}\text { Rule-governed collaborative } \\
\text { problem solving }\end{array}$ \\
\hline \multirow{4}{*}{$\begin{array}{l}\text { I. Working well } \\
\text { with others }\end{array}$} & $\begin{array}{l}\text { - The morality of sympathy: } \\
\text { pure cooperation. }\end{array}$ & $\begin{array}{l}\text { - The morality of fairness: } \\
\text { cooperativeness of } \\
\text { competition. }\end{array}$ \\
\hline & $\begin{array}{l}\text { - Interactional symmetry. Equal } \\
\text { contributions are not so } \\
\text { important. }\end{array}$ & $\begin{array}{l}\text { - Contribution symmetry. Equa } \\
\text { contributions are important. }\end{array}$ \\
\hline & $\begin{array}{l}\text { - Emotional reciprocity is } \\
\text { important. Quality in the close } \\
\text { proximate relations is } \\
\text { important. Social skills are } \\
\text { required. }\end{array}$ & $\begin{array}{l}\text { - Emotional reciprocity is not so } \\
\text { important. Social skills not } \\
\text { required to same degree. }\end{array}$ \\
\hline & $\begin{array}{l}\text { - Ideas are co-constructed } \\
\text { throughout the process. } \\
\text { Collaboration is integral to the } \\
\text { complete process. }\end{array}$ & $\begin{array}{l}\text { - An element of competition is } \\
\text { important. Balancing many } \\
\text { perspectives and conflicting } \\
\text { demands of multiple } \\
\text { participants. }\end{array}$ \\
\hline \multirow[t]{2}{*}{$\begin{array}{l}\text { 2. Cognitive } \\
\text { diversity } \\
\text { (How are } \\
\text { contributions } \\
\text { combined?) }\end{array}$} & $\begin{array}{l}\text { - Not possible to identify } \\
\text { separate contributions. The } \\
\text { degree of individual } \\
\text { contributions may vary } \\
\text { because they are so } \\
\text { intertwined. }\end{array}$ & $\begin{array}{l}\text { - Including all separate } \\
\text { contributions (Either } \\
\text { anonymous or personally } \\
\text { identifiable). More distant and } \\
\text { complete units of } \\
\text { contributions. }\end{array}$ \\
\hline & - Co-ownership of ideas. & $\begin{array}{l}\text { - Stronger ownership to ideas } \\
\text { "my idea and your idea." One } \\
\text { challenge is coping with } \\
\text { negative feelings when "your } \\
\text { idea" is criticized. }\end{array}$ \\
\hline \multirow[t]{2}{*}{$\begin{array}{l}\text { 3. Group } \\
\text { organization }\end{array}$} & $\begin{array}{l}\text { - Emergent self-organizing group } \\
\text { structure. Depends on the } \\
\text { particular contributions in the } \\
\text { process. No preplanned } \\
\text { organization. }\end{array}$ & $\begin{array}{l}\text { - Planned group organization } \\
\text { with shared understanding anc } \\
\text { joint goals. Ideally, everyone } \\
\text { follows the same group rules or } \\
\text { norms. }\end{array}$ \\
\hline & $\begin{array}{l}\text { - Challenge: Not being able to } \\
\text { elaborate on the ideas because } \\
\text { rules need to be followed } \\
\text { strictly. }\end{array}$ & - Challenge: Free riders. \\
\hline
\end{tabular}


Further research should more closely examine what "the ability to work well with others" actually implies in different types of group interaction. Woolley et al. (2015) find that interpersonal skills will be important in this type of problem solving. However, the top solver reports from innovation teams also illustrate that individuals can develop relations during the collaborative activities as they unfold. From this perspective, "the ability to work well together" should not only be analyzed as an individual interpersonal skill (see Chapter 6), but as something that emerges through the interaction itself. The inherent joy in participating may also strengthen group relationships.

Second, cognitive diversity can be achieved in two different ways in collaborative problem solving. On one hand, in elaborative collaboration, it will be part of the immediate co-construction of thoughts. On the other hand, in rule-governed collaboration, the ideas will typically first be proposed as separate individual contributions or ideas before they afterwards are combined into a coherent group solution. In this context, it becomes more challenging to criticize each other's contributions because ideas become more individualized. Collaborators need to learn to control their negative feelings when they are criticized.

Third, group organization is closely related to rule-governed collaboration in that it is organized according to prespecified rules that every group member needs to follow. This will typically become more important as the group size increases in order to ensure that all contributions are equally valuable. The morality of fairness will enforce sanctions on free riders. In contrast, elaborative collaboration will put less emphasis on organizational rules and greater emphasis on conversational flow, interruptions, overlapping talk, and the constant efforts of recombining and modifying ideas. 